\title{
A Hybrid Model for Reasoning about Composed Hardware Systems
}

\author{
E. Thomas Schubert \\ Department of Computer Science \\ Portland State University
}

\begin{abstract}
To formally specify and reason about composed systems, a process algebra is developed that integrates an extended interpreter model. This approach utilizes the interpreter model for device decomposition, while also being able to reason about larger systems that require interdevice communication. By combining these approaches, convenient notations are available to specify and verify both device independent properties (e.g., instruction sets) and device interdependent properties (e.g., communication protocols).
\end{abstract}

\section{Introduction}

Previous approaches to system verification have decomposed systems into several hardware and software layers that may be independently verified $[3,9,11,17]$. Each layer consists of an implementation description and a more abstract specification. The layers are joined, or "stacked", with each implementation description serving as the specification for the next lower layer. The hardware layers of these systems have been modeled as a microprocessor with memory. This simple specification of the hardware has been a convenient abstraction for verifying the correctness of software executing on the hardware base, but by inspection, is not representative of modern hardware implementations. A realistic hardware implementation consists of many different interacting components.

Our approach has developed a framework to formally specify and verify the correctness of the communication between hardware devices. The methodology (formalized within the HOL theorem proving system) allows a hardware system to be decomposed into a set of independently verified devices and provides a logic to specify and reason about the composed, aggregate system behavior. To demonstrate the technique, we present a system with concurrently executing devices that is verified to correctly pass information and coordinate activities. The system consists of a CPU, a memory subsystem with a memory management unit, a direct memory access device, and a bus controller. The remainder of this section will describe the overall approach. Subsequent sections describe the technique's formalization, a system verification example, and a brief conclusion.

\subsection{Formal System Model of Communicating Interpreters}

Formal reasoning about composed hardware systems requires that a device or system be represented as a well-defined mathematical object that denotes the 
important aspects of a system's behavior. To an outside observer, a system appears as a black box. We are interested in a system's behavior over time with respect to a state and environment. An input environment stream is provided and an output environment stream is observed. The observed behavior is typically a function of the inputs. Further, some systems will demonstrate sequential behavior where the outputs are a function of the input history, rather than just the most recent inputs. In this case, it is clear that the system maintains some sort of internal state.

The interpreter model provides an effective framework to describe and reason about device behavior, but the model does not provide sufficient structure to describe the concurrency inherent in composed systems. Several researchers have developed models for describing concurrent systems (e.g., temporal logic of actions, reactive systems). However, much of this work is too abstract to relate to concrete descriptions of programs or hardware. The literature on reactive systems and process algebras also ignores the internal or transformational aspects of a system while focusing on the system/environment interactions. Our model combines transition and reactive models to describe concurrent systems. This approach requires extensions to the interpreter model so that devices are modeled with a state and environment.

The interpreter model views a system as a single entity defined by a set of attributes that includes: a state, an environment, an operation list, and an operation choice function. A hardware system consisting of several devices (each modeled as an interpreter) will also be identified by these attributes. Due to the concurrent behavior of the composed system, the nature of these attributes is considerably more complex than for an individual interpreter. The interpreter model denotes state and environment as a function of time and the operation list as fixed over time. Concurrency will require a formal system model where the system operation list is also a function of time.

The role of the environment is elevated from its secondary status in the interpreter model. The environment is modeled as active and its behavior cannot be explicitly restricted. In a sense, the interplay between a system and its environment is like a game [1]. The environment is an opponent and the system must adapt a strategy that is guaranteed to "win" regardless of what the environment chooses to do. A system verification proof may wish to show that the strategy always succeeds. As in Lamport's model, we assume that the environment may take some number of actions (possibly zero) followed by at most, one action by the system. However, the environment player is not allowed to win by simply not giving the system a chance to play.

There are three general forms in which devices can be combined: shared input and output environments, shared state, and interlinked output environments (see Figure 1). Each model form requires that individual device (top level) interpreters are verified to perform their respective set of operations correctly. To describe a composed system, a combination of these forms may be required. The "glue logic" that links interpreters is provided by a process algebra. 


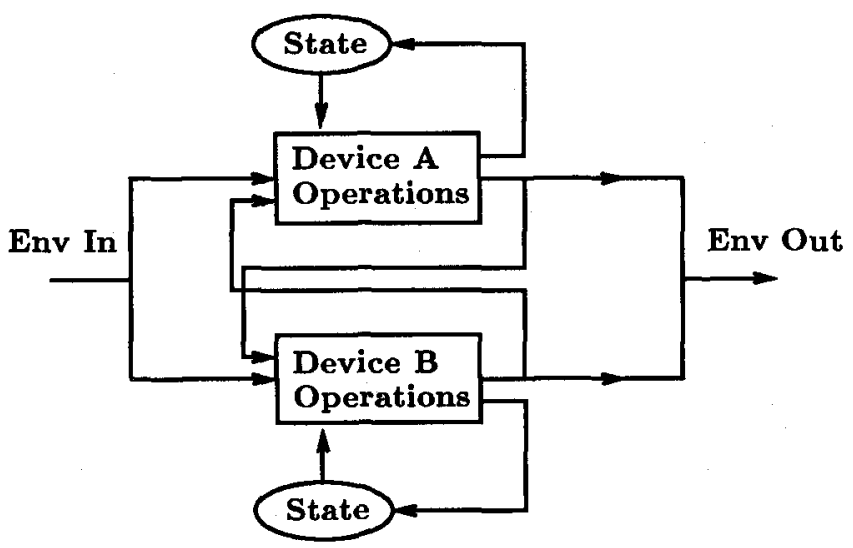

Fig. 1. Linked Environments Black Box Model

\subsection{Process Algebras}

Process algebras, such as CCS, have emerged as a framework to study the interaction of communicating, concurrent processes $[2,7,15]$. While processes are generally thought of as executing programs, hardware systems can also be studied under this framework. Such systems consist of many devices acting independently of each other, but communicating with one another to achieve a mutual goal. The behavior of a system is, in some sense, its entire capability to communicate, or, "what is observable." The behavior of each device can be described as a process and the interaction of devices studied in terms of a process algebra.

All elements of a system, including the medium used to transmit information between devices, are modeled as agents. For example, a hardware system model would not only describe the CPU and memory components as agents, but the bus that connects the two components (the medium) would also be represented by an agent. An agent's potential behavior is described by actions, which may be communications with other agents or independent concurrent actions. Two agents can only communicate only if they perform complementary send and receive actions (e.g.,. $\bar{a}$ and $a$, respectively). Independent actions are represented by the reserved symbol $\tau$ and have no complement. Process algebras also provide agent constants, the most common being $\mathbf{0}$, the "inactive" agent. A summary of the basic agent constructors appears in Table 1.

\begin{tabular}{|lll|}
\hline Operator & Example & \multicolumn{1}{c|}{ Meaning } \\
Prefix & $a . A$ & Action $a$ taken, followed by behavior of agent $A$ \\
Summation & $A+B$ & Behavior of either agent $A$ or agent $B$ \\
Composition & $A \mid B$ & Agent $A$ and agent $B$ operate concurrently \\
Restriction & $A \backslash \ell$ & $\ell$ labeled actions are not visibly performed \\
Relabelling & $A[f]$ & Function $f$ substitutes labels in agent $A$ \\
\hline
\end{tabular}


The basic axioms of CCS are presented in Figure 2. Most variations of CCS incorporate these laws and differ in which equational laws regarding the internal action are included. A brief summary of six such process algebra variations is presented in [6]. Agents with infinite behavior can also be defined with recursive definitions.

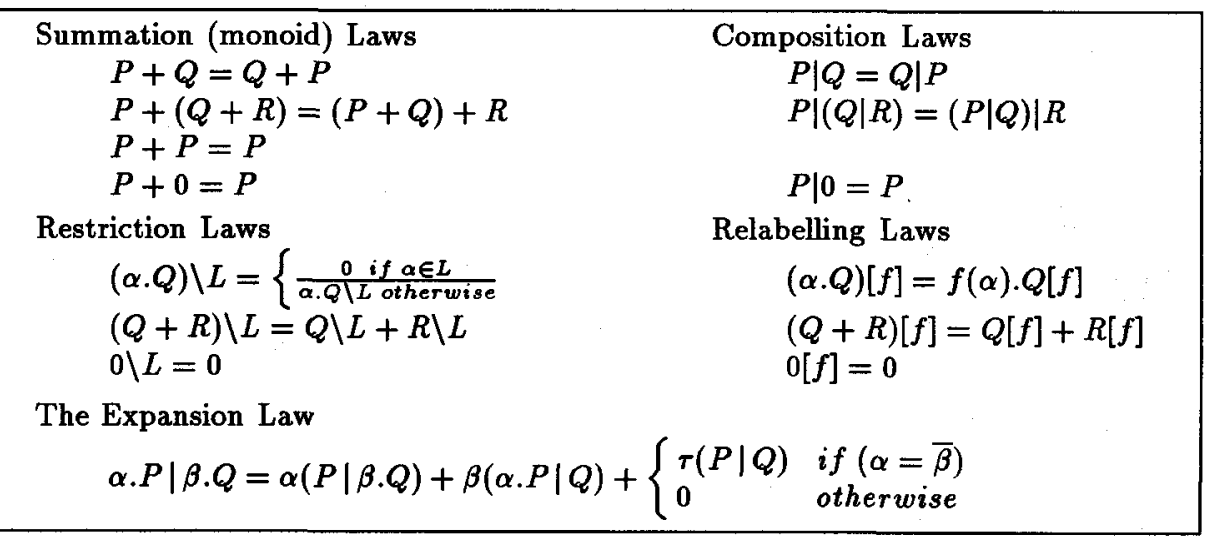

Fig. 2. Basic Axioms of CCS

\subsection{The Hybrid Approach}

The relative benefit (or even presence) of various abstraction mechanisms differs depending on what level of the interpreter hierarchy is being verified. At the lowest levels of the hierarchy, structural and behavioral abstraction mechanisms relate a behavioral description to the structural definition of a device. Proofs between intermediate levels rely primarily on the use of temporal and data abstraction mechanisms. At all levels, the state based approach adopted by the interpreter model seems natural for this problem domain. As the problem domain shifts to verifying a system with multiple devices, we believe a process based approach is more appropriate. Lamport's transition axiom method proposes a state based approach to composing specifications $[1,10]$. However, he points out that a process based approach is equivalent in expressive power. A process based approach seems more natural and frequently, abstract system properties such as security, are more easily expressed using a process based notation [8].

Our approach describes a system as a process algebra term that incorporates a set of individual device interpreters. This approach merges the interpreter (transition) model with a process algebra (reactive) model. Interpreters serve to describe device-private state changes while the process algebra calculus describes the interaction of devices. The system specification must also describe the set of all possible execution interleavings. The static operation selection mechanism provided by the interpreter model assumed that once an action is chosen (e.g., CPU add instruction), the operation runs until completion. In the composed model, operations cannot assume uninterrupted execution; the final result may be different depending on the actions of other devices. To satisfy the arbitrary 
interleaving condition requires that only one critical event occurs per action. This requires that the specification capture intermediate snapshots of interpreter executions when interference is possible.

The process algebra (reactive) model can describe the dynamic nature of device interactions. A process algebra term denotes a function that chooses which interpreter executes next. This in a sense, extends the selection function of the original interpreter model. Greater recognition is also given to the role the environment plays in systems. Process algebra terms capture the specification of environment interactions between devices.

The system is defined as an interpreter function, which given a state and environment, returns a new function, a new state and an output environment. At a given point in time, several devices (interpreters) may be able to act. To verify that the devices can work concurrently, we show that system requirements are satisfied regardless of the interpreter action interleaving.

\section{Mechanization of the Process Algebra}

There has been significant recent interest in mechanizing process algebras in HOL $[4,5,14]$. The process algebra developed here is based on CCS, with extensions to reason about interpreters. Using the HOL system type definition mechanisms, we define an initial algebra for agents, constructed with sequences of actions. HOL's recursive type definition facility [12] automates the process of defining new data types in terms of already existing types. Both new type constants and type constructors (operators) can be defined. Additional (recursive) functions can be defined to operate on the concrete data representation of the type. The properties of new types must be derived by formal proof. This guarantees that the type does not introduce inconsistency into the logic.

Actions: External actions represent enabled (boolean) signals that are part of an interpreter's environment. An external action requires a label that consists of a name and boolean value that denotes whether the action is a send or receive synchronization operation. The private communication action ( $\tau$ action) is replaced by the INTERNAL action. Whereas CCS did not consider what activity was taken by an internal action, internal actions are now tagged with an interpreter that defines changes to private state.

define_type 'action" 'action = IHTERNAL *interpreter I EXTERNAL label'; ;

Agents: The agent data type is constructed from sequences of actions. A preliminary data type consisting of the five CCS type constructors, described in Section 1.2, was developed. This type definition did cover the syntax of the concrete data type. However, we found that the semantics of some of the constructor operators can be defined in terms of others. Additionally, for our purposes, the relabelling operator has not been necessary. Its addition would be simple to add, if warranted by further application of the calculus. By minimizing the number of type operators used for the representation of any type, the complexity of inductive definitions is reduced. 
Sequential Behavior: A term algebra for agents is created through a type definition with a single type constant (IMACTIVE) and two type constructors (PREFIX and SUMM). These elements correspond to their CCS counterparts. An INACTIVE agent (denoted by " 0 " in CCS) cannot communicate with any other entity, and thus, appears to do nothing. The PREFIX operator creates a new agent from an action and an agent. The new agent will first communicate through the action operand and then behave as the agent operand. The SUMM operator creates a new agent that can behave as either of the agent operands. For determining the equivalence of two agents, we would like to define all agents in a normal form with agent terms consisting of only the prefix and summation operators.

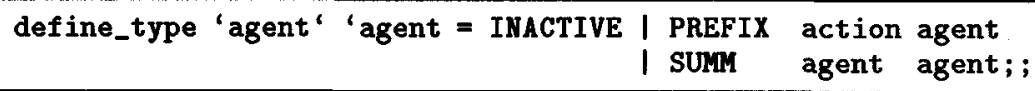

Parallel/Concurrent Behavior: To describe processes that execute in parallel, we adapt a method described in [2]. This technique allows composed, concurrently executing agent expressions to be converted to an equivalent agent expressed only with the prefix and summation operators (as described by the expansion law in Figure 2). We define three mutually recursive functions to replace a composition type constructor (COMM, LMERGE, and COMPOSE). The communication operator, COMM, declares that two agents will communicate if they have complementary, enabled actions (e.g., $a$ and $\bar{a}$ ). The left-merge operator, LMERGE creates a new agent from two agents, such that the new agent must first behave as though only the left agent (first argument) were active. Subsequent behavior is determined by an agent constructed by the composition of the resulting left agent derivative and the original right agent. The compose operator, COMPOSE, creates an arbitrary interleaving of the two agents through use of COMM, LMERGE, and the summation type constructor.

Transition Semantics: Operational semantics for agent expressions can be provided through a labeled transition relation indicating what actions an agent may perform. This relation can be defined using the inductive relation definition package. The inductive relation definition package provides a set of theoremproving tools based on a derived principle of definition in HOL for defining relations inductively by a set of rules [13]. The relation is inductively defined by a collection of such rules to be the least relation closed under all the rules. The defined TRANS predicate, states that for an agent to evolve to another agent, there must be an immediate transition by a prefixed action (e.g., $\alpha . \mathrm{A} \stackrel{\alpha}{\rightarrow}$ A). Symmetric versions of a summation law are provided to indicate that the transition is valid if either SUMM operand agent can make the transition.

\subsection{Agent Equivalence}

Several notions of equivalence between agents can be defined. The kinds of models that satisfy the same equations as the initial algebra will be determined by which notion of equivalence is used. Trace semantic equivalence can be established by defining the meaning of an agent as a set of traces where a trace is 
a sequence of actions. Trace semantics permit fairly broad equivalence classes to be constructed. We are generally interested in a narrower definition of equivalence where two agents are equivalent if an external agent cannot distinguish between the visible behavior (traces) of the two agents. When using the notion of strong equivalence, traces consist of both external and internal actions. For our application, a weaker notion of observation equivalence is sufficient. Observation equivalence states that two equivalent descriptions implement the same external behavior; that is, internal actions cannot be detected by the external agent.

A device specification is an abstraction of the implementation, and thus, will not describe all of the implementation's actions. To show that an implementation satisfies a specification, one-way observation equivalence, is appropriate. Formally, I implements S's behavior if for every action $\alpha$ of S, every $\alpha$-derivative of $\mathbf{S}$ is one-way observation equivalent to some $\alpha$-descendant of $\mathbf{I}$. For the remainder of the paper we will use the term observation equivalence to mean one-way observation equivalence. Observation equivalence can be defined using an inductive relation definition that includes the following rules:

1. Observation equivalence is reflexive: $A \stackrel{o e}{=} A$.

2. Symmetric SUMM terms are observation equivalent: $A+B \stackrel{o e}{=} B+A$.

3. Two agents prefixed with the same action are observation equivalent if the derivative agents (without the prefixed action) are observation equivalent: $A \stackrel{o e}{=} B \Rightarrow \alpha \cdot A \stackrel{o e}{=} \alpha . B$.

4. If an agent $A$ is observation equivalence to an agent $C$ and there is no action for which a transition is possible for an agent $B$ and $C$, then $(A+B)$ and $C$ are observation equivalent: $A \stackrel{\text { oe }}{=} \wedge \wedge(N O T R A N B C) \Rightarrow(A+B) \stackrel{\text { oe }}{=} C$. $A \stackrel{\text { oe }}{=} C \wedge(N O T R A N B C) \Rightarrow(B+A) \stackrel{\text { oe }}{=} C$.

5. If both of the summation agents $(A$ and $B$ ) satisfy observation equivalence with the right-hand side agent $(C)$ then the summation is observation equivalent to $C: A \stackrel{\text { oe }}{=} C \wedge B \stackrel{o e}{=} C \Rightarrow(A+B) \stackrel{\text { ee }}{=} C$.

6. If the right-hand side agent is a summation agent, then the left-hand side agent must satisfy observation equivalence for both of the right-hand side summation agents. This is the symmetric case of the previous rule for a left-hand summation: $A \stackrel{o e}{=} B \wedge A \stackrel{o e}{=} C \Rightarrow A \stackrel{o e}{=}(B+C)$.

7. The final rule requires a side condition that uses an auxiliary reach predicate definition. The term "REACHES A C", is true if $\mathrm{C}$ can be derived from A through only internal actions. This rule states that agents $\mathbf{A}$ and $\mathbf{B}$ are oneway observation equivalent if the internal action derivative of $\mathrm{A}$ is one-way observation equivalent to $\mathrm{B}: A \stackrel{\tau^{*}}{\rightarrow} C \wedge C \stackrel{o e}{=} B \Rightarrow A \stackrel{o e}{=} B$.

\section{System Verification using the Interpreter Calculus}

To demonstrate how system integration can be achieved, we show how several devices can be composed to form a system. The system specification abstracts away details that suggest the system is implemented by several devices. As a first 
example, we will examine the interaction between a modified AVM-1 microprocessor and the memory subsystem. The first example is rather simple system as there is no possibility for device interference. The second example integrates a larger number of devices and integrates interrupt processing among multiple interpreters. With the addition of a direct memory access device (DMA), both the CPU and the DMA may wish to access memory simultaneously. This contention requires that we add a bus controller to the system and redefine the CPU as a set of interpreters. The addition of a DMA also requires the CPU respond to interrupts.

Previous work has constructed examples of how the process algebra can be used to specify and verify the interaction between devices [16]. Communication between devices may require only a single message or a series of messages to be passed between devices. At a low level, the information is passed over a bus using a hardware protocol (e.g., 4-phase handshaking). The types of interactions can be loosely described as belonging to one of the following categories:

Message Passing: Devices pass information asynchronously to one another. Devices may not wait for a sent message to be received and devices may or may not wait (block) for message arrivals.

Process Creation: In the context of hardware, new devices are not "created", but devices may be idle. The process of initiating a new task on an idle processor is analogous to process creation.

Rendezvous: Concurrently-active devices may eventually need to reach a common synchronization point before either can continue execution. Devices will be delayed until all devices reach the rendezvous point.

Remote Procedure Call: This is perhaps the most common form of interaction. A device (e.g., the CPU) requests a service of another device (e.g., the memory) and must wait until the device satisfies the request.

\subsection{CPU/MMU Interaction}

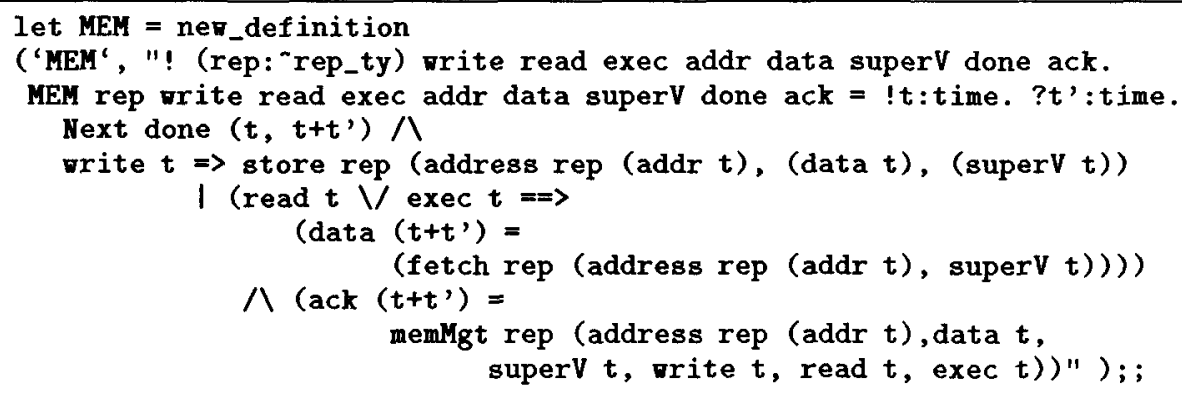

The AVM-1 memory interface specification (above) defines the effect of read and write operations on the state variables memory and data. If a write request is made at time $t$, the memory will reflect this request at some future time $t+t^{\prime}$, otherwise, the memory will remain unmodified. If a read request is made at time $t$, then the data value returned is a function of the memory contents at time $t$. 
Since the actual fetch and store operations are performed by the memory and not the CPU, these functions are abstract and part of the generic representation rep. When combining a CPU and memory, the functions would be instantiated by the concrete memory state manipulation functions. The protection mechanism provided by the MMU makes a distinction between data and instruction memory fetch requests. The exec variable indicates when a memory request is for an instruction code value.

From the perspective of the CPU, all of the parameters to MEM in the specification, are part of the environment. For ease in presentation, we will allow the PREFIX operator to construct an agent from two agents. Thus, $(a+b) . c$ abbreviates $a . c+b . c$.

$$
\begin{aligned}
& \text { done }=a c k+n a c k \\
& \text { cpu_write_request }=(\overline{\text { userMode }}+\tau \cdot \overline{\text { superMode }}) \cdot \overline{\text { write }} . \overline{\text { address }} \text {.done } \\
& \text { cpu_read_request }=(\overline{\text { userMode }}+\tau \cdot \overline{\text { superMode }}) \cdot \overline{\text { read }} \cdot \overline{\text { address }} \text {.done.data } \\
& \text { cpu_exec_request }=(\overline{\text { userMode }}+\tau \cdot \overline{\text { superMode }}) \cdot \overline{\text { exec }} \cdot \overline{\text { address }} \text {.done.data } \\
& \text { CPUtoMEM }=\text { cpu_write_request }+\tau \text {.cpu_read_request }+\tau \text {.cpu_exec_request } \\
& \mathrm{CPU}=\mathrm{CPU} \text { toMEM.CPU }
\end{aligned}
$$

The terms express the actions that the CPU may perform with the use of the internal action $(\tau)$ being particularly significant. The internal action is used to indicate that the CPU will behave in one of several possible ways-with the choice being made internally by the CPU. The process algebra term CPUtoMEM states that the CPU may request memory either write data, read data, or fetch an instruction. Without the use of $\tau$, the term would suggest that any of these events could occur, depending on what an external agent might choose.

Figure 3 pictorially presents the semantic difference when using the $\tau$ action to prefix actions for the case where the CPU chooses to write. The CPU must indicate that process executing is either a user or supervisor process through the $\overline{\text { userMode }}$ or $\overline{\text { superMode }}$ action, respectively. This choice is dependent on the internal state of the CPU. The left-hand side figure shows the case where the CPU presents one output synchronization action to its environment. The CPU will either make a userMode request or a superMode request. The righthand side figure shows the case where both output synchronization events are possible. In this (unrealistic) figure, the CPU indicates that the choice of which synchronization event is up to an external agent.

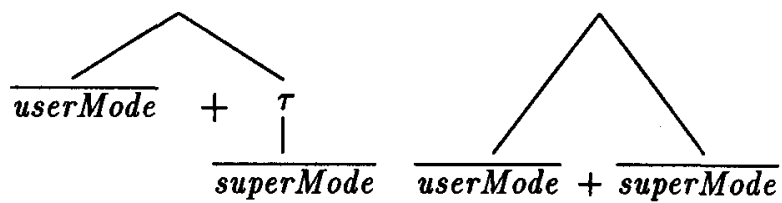

Fig 3. CPU Write Action Example

The MMU interpreter provides seven instructions: superRead, superWrite, superExec, userRead, userWrite, userExec, and updateTblPtr. Unlike the CPU 
(or the DMA described in Section 3.2), operation selection is dependent entirely on the environment. To express the notion that the MMU performs internal actions before responding, the $\tau$ action is inserted before the response is returned. Part of this action may be to update the segment table pointer value or cache. While the MMU is able to accept either a userMode or a superMode communication, the MMU chooses to respond with only $\overline{a c k}$ or $\overline{n a c k}$ communication. The MMU specification also states when the MMU segment table pointer is updated. This action is not relevant to the CPU-MMU interface, so it is expressed as an internal $(\tau)$ action. The specification yields the process algebra terms:

$$
\begin{aligned}
& \text { done }=(\tau \cdot \overline{a c k}+\tau \cdot \overline{n a c k}) \\
& \text { mmu_process_write }=(\text { userMode }+ \text { superMode }) . \text { write.address.data. } \tau . \text { done } \\
& \text { mmu_process_read }=(\text { userMode }+ \text { superMode }) . r e a d . \text { address. } \tau . \text { done. } . \overline{\text { data }} \\
& \text { mmu_process_exec }=(\text { userMode }+ \text { superMode }) . \text { exec.address. } \tau \text {.done. } \overline{\text { data }} \\
& \text { MMUtoCPU }=\text { mmU_process_write }+ \text { mmu_process_read }+ \text { mmu_process_exec } \\
& \text { MMU = MMUtoCPU.MMU }
\end{aligned}
$$

Proof of Correct Composition: The agents CPU and MMU are recursively defined and exhibit an infinite behavior. To show that the composed CPU and MMU communicate correctly, we must show that the system is always able to return to its initial state. It is also necessary to show that progress is made when the CPU initiates a dialogue with the MMU. The proof goal then can be stated as:

1. If either of the CPU actions are enabled, the memory subsystem will engage in communication.

2. The protocol will complete and the system returns to its initial state.

To reason about the finite protocol communication sequences, the recursive behavior of the agents is removed. The recursive agent reference is replaced with an (undefined) agent constant SUCCESS.

To express the goal in a general form, several auxiliary definitions are defined. (ENABLED) is defined to construct a list of all output actions that an agent can perform. A goal predicate definition BECOMES is defined. The predicate states that for all possible enabled output actions, a complement action exists such that a success agent is reached immediately or reached in a descendent.

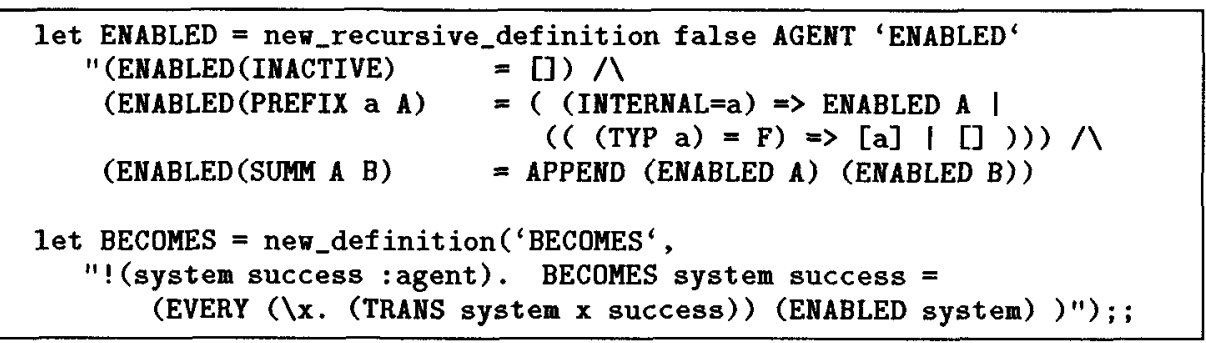

The success agent for the composed MMU-CPU is SUCCESS | SUCCESS. By unwinding the agent definitions and using the TRANS laws, all possible 
paths are found to reach the success agent through internal transitions. The MMU-CPU composed communication proof shows that:

$\vdash B E C O M E S(C P U \mid M M U)$ (SUCCESS | SUCCESS)

\subsection{Multiple Device System Specification}

The inclusion of a DMA controller greatly increases the overall system complexity. A DMA adds interrupt behavior to the system as well as directly interacting with the other system devices. The DMA is programmed to supervise the transfer of a sequence of words between a peripheral device and memory. Upon completion of the transfer, the DMA will generate an interrupt to indicate the transfer has completed. The system described below, places the DMA before the MMU. Thus, DMA memory access requests are validated by the MMU. If the DMA acts as a user process, the MMU may also translate virtual address into real addresses.

Bus Controller Specification: We model the bus controller in a simplified manner, without a fairness property. The controller grants control to either the CPU or DMA and then waits for the controlling device to release the bus. This behavior may be described by the process algebra term:

$$
\begin{aligned}
\text { BUS }= & (\text { CPUreqBus. } \overline{\text { grantCPU }} \cdot \text { CPUrelease.BUS })+ \\
& (\text { DMAreqBus. } . \text { grantDMA } . D M \text { Arelease.BUS })
\end{aligned}
$$

The addition of the bus controller requires a change to the external behavior of the CPU as follows.

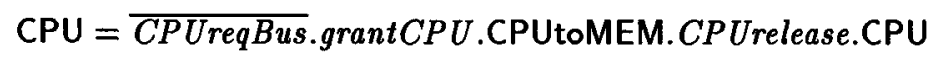

DMA Device Specification: The DMA provides four channels between memory and $1 / O$ devices. The behavior of each channel is determined by a set of registers, which are accessed as memory locations. We will include a DMA with a single write-to-memory channel. The register set for a channel consists of a memory address register, a channel counter register, and a control/status register. The DMA interpreter provides three operations: read DMA register, write DMA register, and service $I / O$ device. The service $\mathrm{I} / \mathrm{O}$ device operation is only available when the channel is enabled. A valid alternative interpreter specification might distinguish between the registers and define unique read and write operations for each of the registers.

To avoid a possible deadlock situation, the DMA agent is defined as two concurrent agents. One agent provides the interpreter operations that provide access to the DMA registers while the other agent describes the service $\mathrm{I} / \mathrm{O}$ device operation. Deadlock might occur if the shared bus resource was being used by the CPU to access a DMA register while the DMA was attempting to obtain the bus in order to write a value to memory. The CPU would not free the bus until the DMA register operation completed and the DMA register operation would not begin until the DMA I/O service operation completedwhich is blocked by the CPU use of the bus. 
DMAreg $=($ write + read $)$. DMAaddress.data. $\overline{\text { done }}$. DMAreg

writeTransfer $=\overline{\text { superMode }} . \overline{\text { write }} . \overline{\text { address }} . \overline{\text { data }}$. done

IOwrite $=\overline{D M A r e q B u s}$. grantDMA. writeTransfer.$\overline{D M A \text { Arelease }}$

DMAio $=i o I n t .1$ Owrite. (DMAio $+\tau . \overline{c p u I n t}$.DMAio)

DMA $=$ DMAreg $\mid \tau$.DMAio

CPU Specification: The potential interference between the CPU and DMA requires a more realistic specification of the CPU. Interference may arise due to the shared use of memory. To avoid multiple critical events during the execution of an instruction, we divide the CPU interpreter specification into the three interpreters: FETCH, DECODE, and EXECUTE. We assume the CPU has an orthogonal instruction set so that each of these interpreters requires at most, one access to memory. The FETCH interpreter either detects an interrupt is pending (from the DMA) or obtains the next instruction from memory. For the present model, we assume that the CPU may not disable interrupt recognition. The DECODE interpreter may obtain an instruction operand from memory. The EXEC interpreter may write one word to memory.

Below we present the process algebra terms to describe the CPU behavior. Rather than assuming that the CPU is driven by an external clock, we assume that the CPU will continuously attempt to execute instructions. Note the use of the $\tau$ action in the agent CPU to indicate that a stage may not access memory.

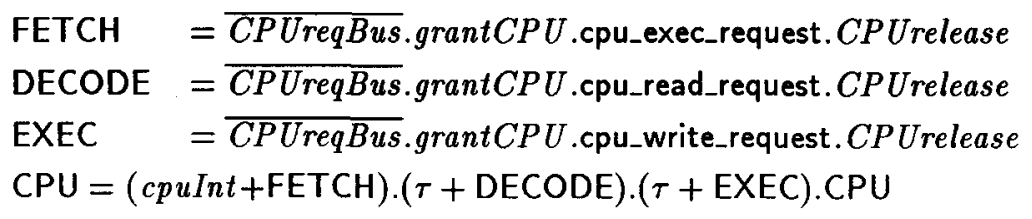

Proof of Correct Composition: We may describe the composed system by the following term, with the system state being the union of all the device states.

$$
\text { System }=\text { CPU } \mid \text { MMU } \mid \text { DMA } \mid \text { BUS }
$$

The correctness proof shows that the composed system is observationally equivalent to a system that abstracts away all the communication between devices. One such proof we show is that the BUS can be abstracted away from the system:

$$
\text { System } \stackrel{o e}{=} \mathrm{CPU}|\mathrm{MMU}| \mathrm{DMA}
$$

\section{Conclusion}

We have presented a framework to formally verify the correctness of communication between composed devices. Previous system verification research has developed vertically verified systems. However, the hardware bases for these systems have been simplistic. Our research is developing a framework to verify a more realistic horizontally verified system. This work demonstrates that CCS is a good choice for describing interdevice implementation-level connections within a computer system. Additional research will expand the calculus and address 
automating the derivation of process algebra expressions from interpreter specifications. Several improvements are being investigated, including an additional type constructor for recursive agents, greater proof support, and automation of the tedious aspects of the proofs.

\section{References}

1. Martin Abadi and Leslie Lamport. Composing specifications. Technical Report 66, Digital Systems Research Center, October 1990.

2. J. C. M. Baeten and W. P. Weijland. Process Algebra. Cambridge University Press, 1990.

3. William R. Bevier, Warren A. Hunt, and William D. Young. Toward verified execution environments. IEEE Symposium on Security and Privacy, 1987.

4. Albert Camilleri, Paola Inverardi, and Monica Nesi. Combining Interaction and Automation in Process Algebra Verification. Lecture Notes in Computer Science No. 494. Springer Verlag, 1991.

5. Albert John Camilleri. Mechanizing CSP trace theory in Higher Order Logic. IEEE Transactions on Software Engineering, 16(9):993-1004, September 1990.

6. R. DeNicola, P. Inverardi, and M. Nesi. Using the axiomatic presentation of behavioral equivalences for manipulating ccs specifications. In International Worshop on Automatic Verification Methods for Finite State Systems, Lecture Notes in Computer Science No. 407, pages 54-67. Springer Verlag, 1989.

7. C.A.R. Hoare. Communicating Sequential Processes. Prentice Hall, 1985.

8. J. L. Jacob. Specifying security properties. In C.A.R. Hoare, editor, Development in Concurrency and Communication. Addision-Wesley, 1990.

9. Jeffrey J. Joyce. Totally verified systems: Linking verified software to verified hardware. In M. Leeser and G. Brown, editors, Hardware Specification, Verification and Synthesis: Mathematical Aspects, Lecture Notes in Computer Science No. 408. Springer Verlag, July 1989.

10. Leslie Lamport. A simple approach to specifying concurrent systems. Communications of the $A C M, 32(1)$, January 1989.

11. David May and David Shepherd. Towards totally verified systems. In Conference on Mathematics of Program Construction, Lecture Notes in Computer Science No. 375. Springer-Verlag, June 1989.

12. Tom Melham. Automating recursive type definitions in higher order logic. In G. Birtwhistle and P.A Subrahmanyam, editors, Current Trends in Hardware Verification and Automated Theorem Proving, pages 341-386. Springer-Verlag, 1989.

13. Tom Melham. A package for inductive relation definitions in HOL, 1991.

14. Tom Melham. A mechanized theory of the $\pi$-calculus in HOL. Technical Report 244, Computer Lab, University of Cambridge, 1992.

15. Robin Milner. Communication and Concurrency. Prentice Hall, 1989.

16. E. Thomas Schubert. A Methodology for the Formal Verification of Composed Hardware Systems. PhD thesis, University of California, Davis, 1992.

17. Phillip J. Windley. The Formal Verification of Generic Interpreters. PhD thesis, University of California, Davis, 1990. 